\title{
Similitude Analysis on Mechanical Parameters of Thin Walled Shells
}

\author{
Gaoming Qin, Haiyang Duan, Zhong Luo, Qingkai Han* \\ School of Mechanical Engineering and Automation, Northeastern University, \\ Shengyang 110004, PRC \\ *qhan@mail.neu.edu.cn (Corresponding author)
}

\begin{abstract}
Keywords: thin walled cylindrical shells, kinetic fundamental parameters, similitude theory, finite element method, scale factors, scaling laws
\end{abstract}

\begin{abstract}
The relationship of the kinetic fundamental parameters for both the prototype and model are derived by employing the finite method. Based on the relations, the scaling laws of the thin walled cylinder for the free vibration are found by applying the similitude transformation to the governing equation. In the absence of the experimental data, the validity of the scaling laws is testified by numerical data. This is done by calculating theoretically the natural frequencies for free vibration of the cylinders. By substituting the model frequencies in the scaling laws, the frequencies of the prototype are obtained. Consequently, the frequencies of the model and prototype are compared. Examples that one end of the thin walled shell is clamped and the other is simply supported show exact agreement.
\end{abstract}

\section{Theoretical solution}

Compared with other mechanical structures, thin walled cylindrical shells have large quantities of merits, such as strong stiffness, light-weight construction etc. Hence, they are widely applied in the areas of aeronautics and astronautics. With lower experience and less time, Employment of similitude theory to establish similarity between prototypes and models in designing thin walled cylindrical shells has become more important. Whereas in contrast, related studies are limited, and concentrated on free vibration response by using equation analysis based on Love Equation or Donnell Equation $^{[1][2]}$. This paper investigated scaling laws associated with kinetic fundamental parameters like mass matrix, stiffness matrix and damping matrix, for thin walled cylindrical shells.

Considering a thin cylindrical shell, Assume the displacements are

$$
\left\{\begin{array}{l}
u=\sum_{m} u_{m}(z, t) \cos m \beta \\
v=\sum_{m} v_{m}(z, t) \sin m \beta . \\
w=\sum_{m} w_{m}(z, t) \cos m \beta
\end{array} .\right.
$$

$u$ is the displacement along the axis of revolution, $v$ is the change of angle $\beta$ of the normals and $w$ is in the radius direction along the radii of curvature.

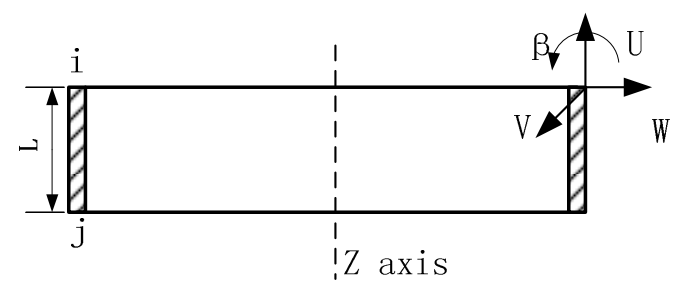

Fig. 1. The ring element

In this paper, ring elements shown in Fig 1 are employed to illustrate the method ${ }^{[3]}$ Let displacements of the element be expressed in the form of interpolation polynomial. 


$$
\left\{\begin{array}{l}
U_{m}(z, t)=\alpha_{1}+\alpha_{2} z \\
V_{m}(z, t)=\alpha_{3}+\alpha_{4} z \\
W_{m}(z, t)=\alpha_{5}+\alpha_{6} z+a_{7} z^{2}+\alpha_{8} z^{3}
\end{array} .\right.
$$

Consequently, Displacements of any point in the ring element can be calculated by those of the node $\mathrm{i}$ and the node $\mathrm{j}$.

$$
\left\{\begin{array}{c}
u_{m}(z, t) \\
v_{m}(z, t) \\
w_{m}(z, t)
\end{array}\right\}=\mathbf{N} \boldsymbol{\delta}_{c n}^{e} .
$$

Eq. 3 is the general case that the shape function of the thin-wall cylindrical shell $\mathbf{N}$ is a matrix of $\zeta$ and $L$, and $\boldsymbol{\delta}_{\mathrm{cn}}^{\mathrm{e}}$ is the column vector to express displacements of two nodes $i$ and $j$.

$$
\begin{aligned}
& \mathbf{N}=\left[\begin{array}{cccccccc}
1-\xi & 0 & 0 & 0 & \xi & 0 & 0 & 0 \\
0 & 1-\xi & 0 & 0 & 0 & \xi & 0 & 0 \\
0 & 0 & 1-3 \xi^{2}+2 \xi^{3} & \left(\xi-2 \xi^{2}+3 \xi^{3}\right) L & 0 & 0 & 3 \xi^{2}-2 \xi^{3} & \left(\xi^{3}-\xi^{2}\right) L
\end{array}\right] \\
& \boldsymbol{\delta}_{c n}^{e}=\left\{u_{n i}(z, t) \quad v_{m i}(z, t) \quad w_{m i}(z, t) \quad \beta_{m i}(z, t) \quad u_{n j}(z, t) \quad v_{n j}(z, t) \quad w_{n j}(\theta, t) \quad \beta_{m j}(\theta, t)\right\}^{\mathrm{T}} \text { and } \quad \xi=\frac{z}{L}
\end{aligned}
$$

In order to obtain the scaling laws composed of scale factors, only one item of each direction is needed, however, they can be applied to the whole displacements $u_{m}(z, t), v_{m}(z, t), w_{m}(z, t)$. The formula can be written like Eq.4, and the strain-displacement relationship for cylindrical shells is Eq.5:

$$
\left\{\begin{array}{l}
u \\
v \\
w
\end{array}\right\}=\left[\begin{array}{cccc}
\cos m \theta & 0 & 0 & 0 \\
0 & \sin m \theta & 0 & 0 \\
0 & 0 & 0 & \cos m \theta
\end{array}\right] \mathbf{N} \boldsymbol{\delta}_{c n}^{e} .
$$

Where $u, v$ and $w$ are the displacements of any point in the ring element. Substituting Eq.4 into Eq. $5^{[4]}$, another useful equation can be obtained.

$$
\left\{\begin{array}{l}
\varepsilon_{z} \\
\varepsilon_{\theta} \\
\varepsilon_{z \theta} \\
k_{z} \\
k_{\theta} \\
k_{z \theta}
\end{array}\right\}=\left[\begin{array}{ccc}
\frac{\partial}{\partial z} & 0 & 0 \\
0 & \frac{1}{a} \frac{\partial}{\partial \theta} & \frac{1}{a} \\
\frac{1}{a} \frac{\partial}{\partial \theta} & \frac{\partial}{\partial z} & 0 \\
0 & 0 & -\frac{\partial^{2}}{\partial z^{2}} \\
0 & -\frac{1}{a^{2}} \frac{\partial^{2}}{\partial \theta^{2}} \\
0 & 0 & -\frac{1}{a} \frac{\partial^{2}}{\partial z \partial \theta}
\end{array}\right]\left[\begin{array}{l}
u \\
v \\
w
\end{array}\right] .
$$

Hence, $\boldsymbol{\varepsilon}=\mathbf{t}_{\mathbf{2}}\left[\mathbf{B}_{1} \cos m \theta+\mathbf{B}_{2} \sin m \theta\right] \mathbf{t}_{\mathbf{1}} \boldsymbol{\delta}_{c n}^{e}$

where $\mathbf{B}_{1}$ and $\mathbf{B}_{2}$ are the matrices of $m$ and $\zeta$. Provided that two similar cylindrical shells have the same circumferential half waves, $\mathbf{B}_{1 \mathrm{~m}}$ and $\mathbf{B}_{2 \mathrm{p}}$ are equivalent respectively with $\mathrm{m}$ referring to the prototype and p referring to the model, since $\zeta_{\mathrm{m}}$ and $\zeta_{\mathrm{p}}$ are dimensionless parameters. 


$$
\begin{aligned}
& \mathbf{B}_{1}=\left[\begin{array}{cccccccc}
-1 & 0 & 0 & 0 & 1 & 0 & 0 & 0 \\
0 & m(1-\xi) & 1-3 \xi^{2}+2 \xi^{3} & \xi-2 \xi^{2}+\xi^{3} & 0 & \xi & 3 \xi^{2}-2 \xi^{3} & -\xi^{2}+\xi^{3} \\
0 & 0 & 0 & 0 & 0 & 0 & 0 & 0 \\
0 & 0 & 12 \xi-6 & 4-6 \xi & 0 & 0 & 12 \xi-6 & 6 \xi-2 \\
0 & 0 & m^{2}\left(1-3 \xi^{2}+2 \xi^{3}\right) & m^{2}\left(\xi-2 \xi^{2}+\xi^{3}\right) & 0 & 0 & m^{2}\left(3 \xi^{2}-2 \xi^{3}\right) & m^{2}\left(-\xi^{2}+\xi^{3}\right) \\
0 & 0 & 0 & 0 & 0 & 0 & 0 & 0
\end{array}\right] \\
& \mathbf{B}_{2}=\left[\begin{array}{cccccccc}
0 & 0 & 0 & 0 & 0 & 0 & 0 & 0 \\
0 & 0 & 0 & 0 & 0 & 0 & 0 & 0 \\
(-1+\xi) m & -1 & 0 & -\xi m & 1 & 0 & 0 & 0 \\
0 & 0 & 0 & 0 & 0 & 0 & 0 & 0 \\
0 & 0 & 0 & 0 & 0 & 0 & 0 & 0 \\
0 & 0 & \left(6 \xi-6 \xi^{2}\right) m & \left(1-4 \xi+3 \xi^{2}\right) m & 0 & 0 & \left(6 \xi^{2}-6 \xi\right) m & \left(3 \xi^{2}-2 \xi\right) m
\end{array}\right] \mathbf{t}_{3} \\
& \mathbf{t}_{1}=\operatorname{diag}\left(1,1, \frac{1}{L}, 1,1, \frac{1}{L}\right) \quad \mathbf{t}_{2}=\operatorname{diag}\left(\frac{1}{L}, \frac{1}{a}, \frac{1}{a}, \frac{1}{L^{2}}, \frac{1}{a^{2}}, \frac{1}{L}\right) \quad \mathbf{t}_{3}=\operatorname{diag}\left(1,1, \frac{a}{L}, 1,1, \frac{a}{L}\right)
\end{aligned}
$$

Stiffness matrices of the local and global coordinates are the same in respect that local and global coordinates are identical except $\mathrm{z}$ direction. In other words, the stiffness matrices can be transformed in a feasible way.

$$
\begin{aligned}
\mathbf{K}^{e} & =\int_{0}^{1} \int_{0}^{2 \pi} \mathbf{t}_{1}^{\mathrm{T}}\left(\mathbf{B}_{1} \cos m \theta+\mathbf{B}_{2} \sin m \theta\right)^{\mathrm{T}} \mathbf{t}_{2}^{\mathrm{T}} \mathbf{D t}_{2}\left(\mathbf{B}_{1} \cos m \theta+\mathbf{B}_{2} \sin m \theta\right) \mathbf{t}_{1} l r d \theta d \xi \\
& =\int_{0}^{1} \mathbf{t}_{1}^{\mathrm{T}}\left(\mathbf{B}_{1}^{\mathrm{T}} \mathbf{t}_{2}^{\mathrm{T}} \mathbf{D t}_{2} \mathbf{B}_{1}+\mathbf{B}_{2}^{\mathrm{T}} \mathbf{t}_{2}^{\mathrm{T}} \mathbf{D t}_{2} \mathbf{B}_{2}\right) \mathbf{t}_{1} \pi l r d \xi
\end{aligned}
$$

where $\mathbf{D}$ is an elastic matrix, it is shown as follows.

$$
\mathbf{D}=\frac{E h}{1-u^{2}}\left[\begin{array}{cccccc}
1 & u & 0 & 0 & 0 & 0 \\
u & 1 & 0 & 0 & 0 & 0 \\
0 & 0 & \frac{1-u}{2} & 0 & 0 & 0 \\
0 & 0 & 0 & -\frac{h^{2}}{12} & -\frac{u h^{2}}{12} & 0 \\
0 & 0 & 0 & -\frac{u h^{2}}{12} & -\frac{h^{2}}{12} & 0 \\
0 & 0 & 0 & 0 & 0 & -\frac{(1-u) h^{2}}{12}
\end{array}\right]
$$

Note that the elements in the 3 rd and 6th row of $\mathbf{B}_{1}$ are all zeros, while the rows of $\mathbf{B}_{2}$ that are not zeros are only the $3 \mathrm{rd}$ and 6 th rows. $\mathrm{K}^{\mathrm{e}}$ can thus be interpreted as anther form.

$$
\mathbf{K}^{e}=\int_{0}^{1} \mathbf{t}_{1}^{\mathrm{T}} \mathbf{B}^{\mathrm{T}} \mathbf{t}_{2}^{\mathrm{T}} \mathbf{D} \mathbf{t}_{2} \mathbf{B} \mathbf{t}_{1} \pi l r d \xi
$$

Finally, the mass matrix $\mathbf{M}^{\mathrm{e}}$, the stiffness matrix $\mathbf{K}^{\mathrm{e}}$ and the damping matrix $\mathbf{C}^{\mathrm{e}}$ can be extrapolated as follows.

$$
\begin{aligned}
& \mathbf{M}^{e}=\int_{0}^{1} \int_{0}^{2 \pi} \mathbf{N}^{T} \rho h \mathbf{N} l r d \theta d \xi=\pi \rho h l a \int_{0}^{1} \mathbf{N}^{T} \mathbf{N} d \xi \\
& \mathbf{K}^{e}=\int_{0}^{1} \mathbf{t}_{1}^{\mathrm{T}} \mathbf{B}^{T} \mathbf{t}_{2}^{\mathrm{T}} \mathbf{D} \mathbf{t}_{2} \mathbf{B t} \pi l r d \xi=\pi l a \int_{0}^{1} \mathbf{t}_{1}^{\mathrm{T}} \mathbf{B}^{T} \mathbf{t}_{2}^{\mathrm{T}} \mathbf{D} \mathbf{t}_{2} \mathbf{B} \mathbf{t}_{1} \pi l r d \xi \\
& \mathbf{C}^{e}=\int_{0}^{1} \int_{0}^{2 \pi} \mathbf{N}^{\mathrm{T}} v h \mathbf{N} l r d \theta d \xi=\pi v h l a \int_{0}^{1} \mathbf{N}^{\mathrm{T}} \mathbf{N} d \xi
\end{aligned}
$$

The similitude relationships of $\mathbf{N}$ between the model and prototype can be easily found.

$$
\mathbf{N}_{\mathrm{m}}=\mathbf{N}_{\mathrm{p}} \mathbf{T} \cdot
$$


where $\mathbf{T}_{1}$ is the diagonal matrix. Above all, the scaling laws of mass matrices and damping matrices for both the prototype and model can be written:

$$
\begin{aligned}
& \mathbf{M}^{e}=\mathbf{T}_{1}^{\mathrm{T}} C_{\rho} C C_{a} C_{h} \mathbf{M}_{\mathrm{p}}^{e} \mathbf{T}_{1} . \\
& \mathbf{C}^{e}=\mathbf{T}_{1}^{\mathrm{T}} C_{a} C_{L} C_{h} C_{\mu} \mathbf{C}_{\mathrm{p}}^{e} \mathbf{T}_{1} .
\end{aligned}
$$

$\mathbf{T}_{1}$ describes the relationships between the prototype and the model.

$$
\mathbf{T}_{1}=\operatorname{diag}\left(1,1,1, \frac{L_{\mathrm{m}}}{L_{\mathrm{p}}}, 1,1,1, \frac{L_{\mathrm{m}}}{L_{\mathrm{p}}}\right) \text { and } \mathbf{T}_{2}=\operatorname{diag}\left(\frac{L_{\mathrm{p}}}{L_{\mathrm{m}}}, \frac{a_{\mathrm{p}}}{a_{\mathrm{m}}}, \frac{a_{\mathrm{p}}}{a_{\mathrm{m}}}, \frac{L_{\mathrm{p}}^{2}}{L_{\mathrm{m}}^{2}}, \frac{a_{\mathrm{p}}^{2}}{a_{m}^{2}}, \frac{L_{\mathrm{p}}^{2}}{L_{\mathrm{m}}^{2}}\right)
$$

The stiffness of the prototype and model can be written respectively:

$$
\begin{aligned}
& \mathbf{K}_{\mathrm{p}}^{e}=\pi l a \int_{0}^{1} \mathbf{t}_{1}^{\mathrm{T}} \mathbf{B}_{m}^{\mathrm{T}} \mathbf{t}_{2}^{\mathrm{T}} \mathbf{D}_{\mathrm{p}} \mathbf{t}_{2} \mathbf{B}_{\mathrm{m}} \mathbf{t}_{1} d \xi . \\
& \mathbf{K}_{\mathrm{m}}^{\mathrm{e}}=\pi l a \int_{0}^{1} \mathbf{T}_{1} \mathbf{t}_{1}^{\mathrm{T}} \mathbf{B}_{p}^{\mathrm{T}} \mathbf{t}_{2}^{\mathrm{T}} \mathbf{T}_{2}^{\mathrm{T}} \mathbf{T}_{2}^{\mathrm{T}} \mathbf{D}_{m} \mathbf{T}_{2} \mathbf{t}_{2} \mathbf{B}_{p} \mathbf{t}_{1} \mathbf{T}_{1} d \xi .
\end{aligned}
$$

If $\mathbf{K}_{\mathrm{m}}{ }^{\mathrm{e}}$ and $\mathbf{K}_{\mathrm{p}}{ }^{\mathrm{e}}$ have similar relationships, $\mathbf{T}_{2}{ }^{\mathrm{T}} \mathbf{D}_{m} \mathbf{T}_{2}$ has to be the same with or several times by a constant than $\mathbf{D}_{\mathrm{p}}$, so does $\mathbf{B}_{\mathrm{m}}$ and $\mathbf{B}_{\mathrm{p}}$. If $\mathrm{C}_{a}=\mathrm{C}_{L}, \mathrm{C}_{\mathrm{m}}=1$, through a set of simplifications, we can obtain:

$$
\mathbf{T}_{2}^{\mathrm{T}} \mathbf{D}_{m} \mathbf{T}_{2}=\frac{C_{E}}{C_{h}} \mathbf{D}_{\mathrm{p}}
$$

if $\mathrm{C}_{a}=\mathrm{C}_{L}$, then $\mathbf{K}_{m}^{e}=\mathbf{T}^{\mathrm{T}} C_{E} C_{a} \mathbf{K}_{\mathrm{p}}^{e} \mathbf{T}^{\mathrm{T}}$

The mass matrix $\mathbf{M}$, the stiffness matrix $\mathbf{K}$ and the damping matrix $\mathbf{C}$ are integrated by those of the elements in sequence of element numbers. Hence:

$$
\begin{gathered}
\mathbf{M}=\sum \mathbf{M}^{e}=\mathbf{T}^{\mathrm{T}} \mathbf{M}_{0} \mathbf{T} \\
\mathbf{K}=\sum \mathbf{K}^{e}=\mathbf{T}^{\mathrm{T}} \mathbf{K}_{0} \mathbf{T} \\
\mathbf{C}=\sum \mathbf{C}^{e}=\mathbf{T}^{\mathrm{T}} C_{0} \mathbf{T}
\end{gathered}
$$

where $\mathbf{T}=\operatorname{diag}\left(\mathbf{T}_{\infty 0}, \mathbf{T}_{00} \ldots . ., \mathbf{T}_{\infty 0}\right)$ and $\mathbf{T}_{00}=\operatorname{diag}\left(1,1,1, \frac{L_{\mathrm{m}}}{L_{\mathrm{p}}}\right)$

Eq. 1 yields that $\mathrm{n}$ is determined by $u_{\mathrm{m}}(s, t)$, and the necessary condition that the mode shapes of the model and prototype are similar is $n_{\mathrm{m}}$ and $n_{\mathrm{p}}$ have to be identical. Accordingly, the scaling laws concerning $\mathbf{M}, \mathbf{K}$ and $\mathbf{C}$ are obtained in the following form.

$$
\begin{aligned}
& \mathbf{M}_{\mathrm{m}}=C_{\rho} C_{l}^{3} \mathbf{T}^{T} \mathbf{M}_{p} \mathbf{T} \\
& \mathbf{K}_{m}=C_{l} C_{E} \mathbf{T}^{T} \mathbf{K}_{p} \mathbf{T} \\
& \mathbf{C}_{\mathrm{m}}=C_{v} C_{l}^{3} \mathbf{T}^{T} \mathbf{C}, \mathbf{T} \\
& C_{m}=1, C_{n}=1, C_{u}=1
\end{aligned}
$$

\section{Similitude of natural frequencies and mode shapes}

The governing equation without damping is. ${ }^{[6]}$

$$
\mathbf{M} \ddot{\mathbf{\delta}}+\mathbf{K} \boldsymbol{\delta}=0
$$

the solution is assume to be $\boldsymbol{\delta}=\tilde{\boldsymbol{\delta}} \sin \omega t$, Substituting the solution in Eq. 18 results.

$$
\left(\mathbf{K}-\omega^{2} \mathbf{M}\right) \tilde{\boldsymbol{\delta}}=0
$$

Utilizing Eq.14 to Eq.17and Eq.19,we can express the scaling laws related to frequencies.

$$
C_{\omega}=\frac{1}{C_{l}} \sqrt{\frac{C_{E}}{C_{\rho}}}
$$


Since the governing equations are equivalent and the boundary conditions are the same between models and prototypes, the mode shapes are certain to be the same.

Table 1 Geometrical parameters of the thin walled cylindrical shells

\begin{tabular}{|l|l|l|l|l|l|l|}
\hline & $\begin{array}{l}\text { Length } 1 \\
(\mathrm{~m})\end{array}$ & $\begin{array}{l}\text { Thickness } \\
(\mathrm{m})\end{array}$ & $\begin{array}{l}\text { outer diameter } D \\
(\mathrm{~m})\end{array}$ & $\begin{array}{l}\text { Young's modulus } \\
\mathrm{E} / \mathrm{Pa}\end{array}$ & $\begin{array}{l}\text { Poisson's ratio } \\
\mu\end{array}$ & $\begin{array}{l}\text { Density } \rho \\
\mathrm{Kg} / \mathrm{m}^{3}\end{array}$ \\
\hline Prototype & 0.1 & 0.272 & 0.002 & $1.09 \times 10^{11}$ & 0.34 & 4440 \\
\hline Model 1 & 0.05 & 0.136 & 0.001 & $1.09 \times 10^{11}$ & 0.34 & 4440 \\
\hline Model 2 & 0.025 & 0.136 & 0.001 & $1.09 \times 10^{11}$ & 0.34 & 4440 \\
\hline Model3 & 0.05 & 0.068 & 0.001 & $1.09 \times 10^{11}$ & 0.34 & 4440 \\
\hline Model 4 & 0.05 & 0.136 & 0.002 & $1.09 \times 10^{11}$ & 0.34 & 4440 \\
\hline Model 5 & 0.05 & 0.136 & 0.001 & $1.09 \times 10^{11}$ & 0.34 & 8880 \\
\hline Model6 & 0.05 & 0.136 & 0.001 & $2.18 \times 10^{11}$ & 0.34 & 4440 \\
\hline Model 7 & 0.05 & 0.136 & 0.001 & $1.09 \times 10^{11}$ & 0.17 & 4440 \\
\hline
\end{tabular}

Validation by FEM

One prototype and several models are designed to verity the correctness of Eq.9, Table 1 contains the parameters of the prototype and models. The boundary condition is one end is clamped and the other is simply supported. Numerical experiments are done.

Observing mode shapes of different orders, the number of axial half waves are all 1, whreas the number of circumferential waves is displayed in Table 2 , we can see that only model 2, model 5 and model 6 have similar relationships with the prototype. This extrapolates that if identical parameters of two thin walled cylindrical shells can be converted to each other, the geometries must have the equivalent scale factors and $u$ must be identical, while the material properties $E$ and $\rho$ are not constraint.

Table 2 The five orders of natural frequencies of scaled cylinder shells

\begin{tabular}{lccccc}
\hline & 1 & 2 & 3 & 4 & 5 \\
\hline The prototype & 904.11 & 914.83 & 942.47 & 976.06 & 1019.0 \\
Model 1 & 1808.2 & 1829.7 & 1884.9 & 1952.1 & 2038.0 \\
Scale factor & 2.0000 & 2.0000 & 2.0000 & 2.0000 & 2.0000 \\
Model 2 & 3589.8 & 3614.4 & 3634.4 & 3707.3 & 3747.1 \\
Scale factor & 3.9705 & 3.9509 & 3.8563 & 3.7982 & 3.6772 \\
Model 3 & 2577.1 & 2603.3 & 2991.4 & 3016.3 & 3634.2 \\
Scale factor & 2.8504 & 2.8457 & 3.1740 & 3.0903 & 3.5664 \\
Model 4 & 2517.5 & 2569.3 & 2632.6 & 2792.7 & 2889.2 \\
Scale factor & 2.7845 & 2.8085 & 2.7933 & 2.8612 & 2.8353 \\
Model 5 & 1278.6 & 1293.8 & 1332.9 & 1380.4 & 1441.1 \\
Scale factor & 1.4142 & 1.4143 & 1.4143 & 1.4143 & 1.4142 \\
Model 6 & 2557.2 & 2587.5 & 2665.7 & 2760.7 & 2882.1 \\
Scale factor & 2.8284 & 2.8284 & 2.8284 & 2.8284 & 2.8284 \\
Model 7 & 1816.8 & 1855.0 & 1879.0 & 1997.7 & 2017.9 \\
Scale factor & 2.0095 & 2.0277 & 1.9937 & 2.0467 & 1.9803 \\
\hline
\end{tabular}




\section{Conclusions}

The similitude on kinetic fundamental parameters for thin walled cylindrical shells has been investigated in this paper, and simple scaling laws of natural frequencies and mode shapes emerge, which have been found in an excellent agreement with numerical simulations. The sufficient and necessary conditions for two similar cylindrical shells are that their geometrical sizes must be proportional to each others.

Table 3 Circumferential waves of thin walled cylinderical shells

\begin{tabular}{lllllll}
\hline & $1^{\text {st }}$ order & $2^{\text {nd }}$ order & $3^{\text {rd }}$ order & $4^{\text {th }}$ order & $5^{\text {th }}$ order \\
\hline The prototype & 9 & 8 & 10 & 7 & 11 \\
Model & 1 & 9 & 8 & 10 & 7 & 11 \\
Model & 2 & 10 & 11 & 9 & 12 & 8 \\
Model & 3 & 5 & 6 & 7 & 4 & 8 \\
Model & 4 & 7 & 6 & 8 & 5 & 9 \\
Model & 5 & 9 & 8 & 10 & 7 & 11 \\
Model & 6 & 9 & 8 & 10 & 7 & 11 \\
Model & 7 & 9 & 8 & 10 & 7 & 11 \\
\hline
\end{tabular}

\section{Acknowledgment}

This work was supported by National Science Foundation of China (51105064), Postdoctoral Science Foundation of China (20100481200), and Fundamental Research Funds for the Central Universities of China (N100403007).

\section{References}

[1] J. Rezaeepazhand, G..J.Simitses, Scale models for laminated cylindrical shells subjected to axial compression, J. Composite Structures 34 (1996) 371-379

[2] J. Rezaeepazhand, G..J.Simitses, Design of scaled down models for predicting shell vibration response. Journal of Sound and Vibration 195(2), 301-311

[3] Giancarlo Genta, Dynamics of rotating systems, Springer Science and Business Media Inc, New York, 2005

[4] Werner Soedel, Vibrations of shells and plates, third ed., Marcel Dekker Inc, New York, 2004

[5] Boris Krayterman, Gajanan M.Sabnis, Similitude Theory: Plates and shells Analysis, J. Journal of Engineering Mechanics.110 (9)1247-1263

[6] Larry J.Segerlind, Applied finite element analysi, second ed., John Wiley and Sons, New York, 1984. 